\title{
First report of Fusarium verticillioides causing damping-off of corn in Taiwan
}

\author{
Yun-Ching Sun ${ }^{1} \cdot$ Jhu-Yin Siao ${ }^{1} \cdot$ Chien-Jui Huang $^{1}$ (D) \\ Received: 29 March 2019 / Accepted: 23 July 2019 / Published online: 1 August 2019 \\ (C) Società Italiana di Patologia Vegetale (S.I.Pa.V.) 2019
}

Keywords Maize $\cdot$ Fusarium verticillioides $\cdot$ Damping-off

Corn seedlings (Zea mays cv. Bright Jean) showing dampingoff symptoms with the disease incidence of $40 \%$ were observed in the nursery in Chiayi City, Taiwan in 2018. The infected seedlings showed weak, stunting growth and finally died. A fungus was consistently isolated from the blighted seedlings, and two representative isolates, $\mathrm{CF} 1$ and CF2, were obtained. The isolates formed salmon-orange colonies on potato dextrose agar. The microconidia were oval or fusiform with zero to two septate and measured $6.9 \pm 0.8(5.0-8.3) \times$ $2.9 \pm 0.4(2.2-3.8) \mu \mathrm{m}$. The isolates were further identified by sequencing of the internal transcribed spacer (ITS) region of rDNA and partial sequences of the translation elongation factor 1-alpha (TEF1) (O'Donnell et al. 1998). The sequences were deposited in GenBank (MK649932-MK649933 and MK654901-MK654902). In BLASTn searches, the ITS sequences of the isolates showed $100 \%$ identity with Fusarium verticillioides CBS 218.76 (X94166). The TEF1 sequences of the isolates showed $99.8 \%$ and $99.5 \%$ identity with that of $F$. verticillioides CBS 218.76 (KF499582), indicating that both isolates are $F$. verticillioides. The isolate $\mathrm{CF} 1$ was deposited as BCRC FU31195 in Bioresource Collection and Research Center, Taiwan. To fulfill Koch's postulates, surface sterilized corn seeds (cv. Bright Jean) were immersed in a conidial suspension of the isolate CF1 $\left(2.5 \times 10^{5}\right.$ conidia/ $\mathrm{ml}$ ) or sterile water (control) for $48 \mathrm{~h}$ at $25^{\circ} \mathrm{C}$. Inoculated seeds were dried, planted in sterile potting mix in plastic pots and grown for two weeks. The artificially inoculated seedlings showed damping-off symptoms, which were indistinguishable from the natural infections, whilst the control seedlings

Yun-Ching Sun and Jhu-Yin Siao contributed equally to this work.

Chien-Jui Huang

chienjui.huang@mail.ncyu.edu.tw

1 Department of Plant Medicine, National Chiayi University, No. 300, Syuefu Rd., Chiayi City 60004, Taiwan remained symptomless. The same fungus was repeatedly isolated from the symptomatic seedlings. Before this study, seedborne $F$. moniliforme was once reported to be associated with poor growth of corn in Taiwan, but its pathogenicity was not proven ( $\mathrm{Li}$ and $\mathrm{Wu}$ 1986). Since former $F$. moniliforme includes, in addition to $F$. verticillioides, several current fungal species, being at least three common in maize (Seifert et al. 2003), this is the first report confirming $F$. verticillioides as the causal agent of corn damping-off in Taiwan.

Acknowledgements This work was financed by the Ministry of Science and Technology (MOST, grant numbers 107-2311-B-415-004 and 1072321-B-002-056), Taiwan, R.O.C.

\section{Compliance with ethical standards}

Conflict of interest The authors declare that they have no conflict of interest.

Research involving human participants and/or animals The authors declare that no human participants and animals were involved in this study.

Informed consent This manuscript is new and not being considered elsewhere. All authors have approved the submission of this manuscript.

\section{References}

Li SY, Wu WS (1986) Significance and control of seed-borne Fusarium moniliforme of corn. Plant Prot Bull 28:191-202

O'Donnell K, Kistler HC, Cigelnik E, Ploetz RC (1998) Multiple evolutionary origins of the fungus causing Panama disease of banana: concordant evidence from nuclear and mitochondrial gene genealogies. Proc Natl Acad Sci U S A 95:2044-2049

Seifert KA et al (2003) The name Fusarium moniliforme should no longer be used. Mycol Res 107:641-644

Publisher's note Springer Nature remains neutral with regard to jurisdictional claims in published maps and institutional affiliations. 